\title{
Route optimization in the removal of radiation hazards
}

\author{
A. N. Sesekin ${ }^{1,2}$, O. L. Tashlykov ${ }^{1}$, S. Ye. Shcheklein ${ }^{1}$ \\ \& A. G. Chentsov ${ }^{1,2}$ \\ ${ }^{1}$ Ural Federal University, Russia \\ ${ }^{2}$ Institute of Mathematics and Mechanics, UB of RAS, Russia
}

\begin{abstract}
Route optimization is one of the types of jobs peculiar to nuclear facilities. For example, route optimization can be used as a way to minimize the radiation dose to personnel during the dismantling of dangerous radiation equipment. The sequence of jobs affects the total dose exposure of personnel. Also, in the solution of problems there are additional constraints in the form of precedence conditions. These conditions are due to technological constraints on the sequence of dismantling. This problem is similar to the well-known traveling salesman problem, but unlike the classic traveling salesman problem, the cost function depends on the list of outstanding tasks. A version of the method of dynamic programming has been designed to solve this problem and the appropriate software has been created. The developed software to provide estimates reduces personnel exposure in the optimization sequence of dismantling radioactive equipment.
\end{abstract}

Keywords: radiation hazardous objects, dismantling equipment, route optimization.

\section{Introduction}

Any energy technology has a certain danger, namely the possibility of harmful effects on humans and the environment. It carries a threat to the health and life of the operating personnel and the public. Nuclear technology has a specific danger, needing a specific approach to ensure the safety of its use, along with the dangers of other technologies. 
Nuclear radiation of various kinds (gamma, beta, alpha, neutron) is a specific feature of the use of nuclear energy (nuclear technology).

In its present form, the fundamental principles of safety, including radiation safety are detailed in the requirements and recommendations of the ICRP [1]. Principles of Radiation Safety (justification, normalization, optimization, protection of future generations and the environment) are well known, as they concern all citizens, not just professionals.

The principle of optimization of radiation protection should apply to all exposure situations, subject to the limitations of individual doses and radiation risks. In particular, the document recommends a reduction of dose and risk for planned exposure situations [1].

There are various ways to reduce radiation exposure of personnel; system decontamination (i.e. cleaning the interior of the primary system by chemicals and thus removing activity), robotic systems and system shielding can be used to reduce radiation exposure of personnel. Route optimization occupies a special place among the possible ways to minimize the planned radiation exposure in an emergency and existing exposure situation professionals conducting work in conditions of inhomogeneous radiation fields. We show further examples of the effectiveness of routing optimization.

For several years in the Urals Federal University, in the Department of "Applied Mathematics" and "Nuclear Power Plants and Renewable Energy" in collaboration with the Institute of Mathematics and Mechanics, Ural Branch of the Russian Academy of Sciences, carried out the work on the construction methods and algorithms for solving the routing optimization, applicationoriented in applications of nuclear energy.

Initial data for optimizing movement in radiation fields can serve as the data obtained as a result of radiation survey area, or simulation of radiation fields using specialized software.

\section{The problem of optimizing the sequence of dismantling of radiation-contaminated equipment to minimize exposure to personnel}

The problem has important social value in terms of minimizing the collective radiation dose. So the total dose personnel during dismantling of one block of RBMK-1000 is more than $100 \mathrm{man} \cdot \mathrm{Sv}$, and labour costs are about 1.5 million man'hours [2].

Objects (pipes, equipment, etc.) with varying degrees of radioactivity are in separate rooms and boxes of decommissioning reactors. Efforts to dismantle each of these objects are different. The total radiation dose received by workers in dismantling works indoors or on the platform will depend on the time of their stay in the radiation fields produced by radiation-hazardous facilities; the emission intensity of each source.

Radiation fields generated by individual elements of radiation dangerous equipment, which should be dismantled. Parameters of the radiation field are 
changed by the removal of the individual radiation hazardous objects. The authors have developed algorithms for constructing the Bellman function and have carried out the software implementation procedure of dynamic programming [3-5] for solving the problem of minimizing the total dose at work on dismantling the nuclear power output from the operation. The problem under consideration differs from the known traveling salesman problem in that the cost function (the current intensity of the radiation) which depends on the list are not dismantled elements.

Let $\alpha=\alpha(1), \alpha(2), \ldots, \alpha(N)$ be a permutation [6] of $\overline{1, N}$. This permutation defines the sequence of dismantling radiation hazardous elements. The total dose of irradiation when removing the $N$ objects in a sequence $\alpha(1), \alpha(2), \ldots, \alpha(N)$ assuming that the distance factor is not taken into account, is given by

$$
\begin{aligned}
E & =t_{\alpha(1)}\left(\dot{H}_{\alpha(1)}+\dot{H}_{\alpha(2)}+\ldots+\dot{H}_{\alpha(N)}\right)+t_{\alpha(2)}\left(\dot{H}_{\alpha(2)}+\dot{H}_{\alpha(3)}+\ldots\right. \\
& \left.+\dot{H}_{\alpha(N)}\right)+t_{\alpha(2)}\left(\dot{H}_{\alpha(2)}+\dot{H}_{\alpha(3)}+\ldots+\dot{H}_{\alpha(N)}\right)+\ldots+t_{\alpha(N)} \dot{H}_{\alpha(N)} .
\end{aligned}
$$

Here $\dot{H}_{\alpha(i)}$ is the power of the radiation dose to create $\alpha(i)$ elements; $t_{\alpha(i)}$ is the time of dismantling of $i$-s element $\left(t_{\alpha(i)}\right.$ in this problem is independent of the object later dismantled because of travel time between objects neglect). From (1) we see that the intensity of the first object after removing it ceases to affect the total radiation dose. A similar situation occurs with other dismantled facilities.

Now we consider the simplest example of finding the optimal sequence of dismantling radioactive facilities. We assume that in a room is four objects that are to be dismantled. To simplify the calculations we do not consider the impact on the dose of the distance from the radiation source to the worker. We can see the data for the calculation in table 1 .

Table 1: Initial data for calculation.

\begin{tabular}{|l|c|c|c|c|}
\hline \multicolumn{1}{|c|}{ Object number } & 1 & 2 & 3 & 4 \\
\hline The dose rate of the object. relative units/hour & 2 & 3 & 1.5 & 1.5 \\
\hline $\begin{array}{l}\text { Time spent on dismantling the object radiation. } \\
\text { hour }\end{array}$ & 0.2 & 0.3 & 0.7 & 0.5 \\
\hline
\end{tabular}

It is known that there are $N$ ! options of bypassing of $N$ objects for solving the problem of routing optimization. Therefore, for this simple example (four objects in the room) there are 24 variants bypass route (fig. 1).

The calculations found two optimal dismantling sequences corresponding to the minimum dose ( $\mathrm{E}=5.95$ relative unites): $2-1-4-3$ and 1-2-4-3.

In fig. 2 we can see the impact of the radiation fields produced by objects in the dismantling process in the sequence 2-1-4-3.

Maximum dose (in the worst-case scenario) is 11.45 relative units dismantling sequence 4-3-1-2. 


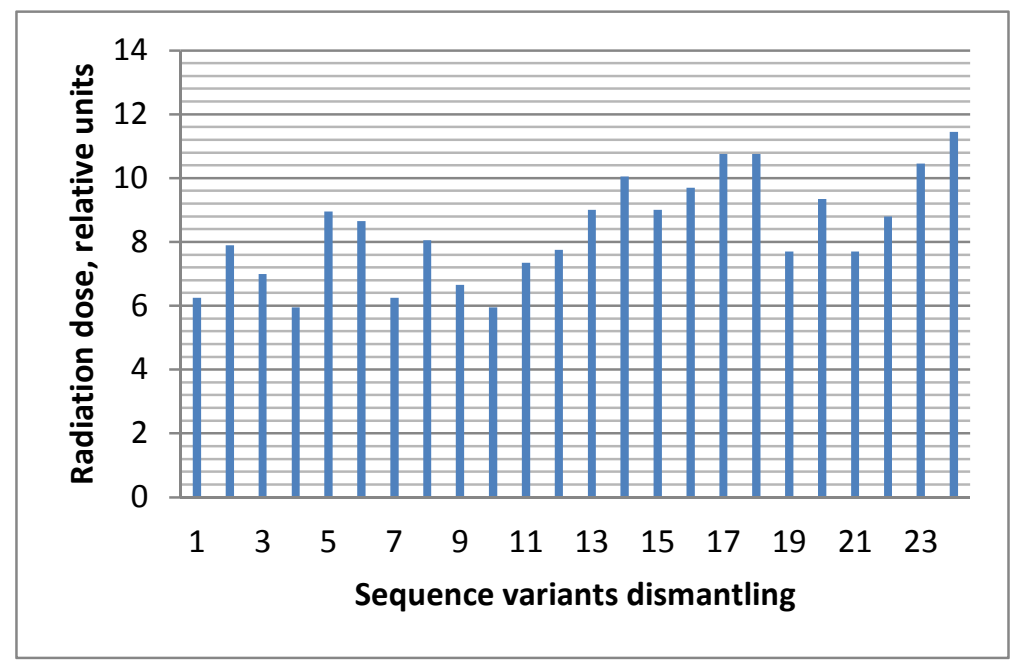

Figure 1: Exposure dose of personnel for possible sequence variants dismantling four radioactive objects in the room.

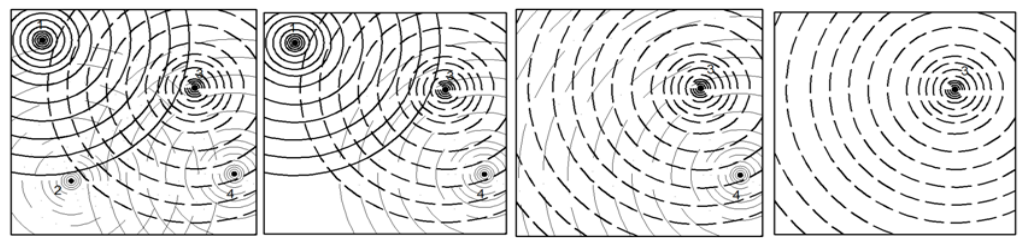

Figure 2: $\quad$ Scheme effects of radiation fields produced by objects in the room during dismantling.

\section{Mathematical formulation of the problem}

Let $N$ be a natural number, $N>2$. This number shows us how many objects we need to dismantle. We will consider the mapping

$$
(i, j, K) \rightarrow c_{i, j}[K]: \overline{0, N} \times \overline{1, N} \times \hbar \rightarrow[0, \infty[.
$$

This map gives the radiation dose received by personnel in the transition from object $i$ to $j$. The map also characterized the radiation dose obtained under dismantling the object $j$ on condition that $K$ is a set of not dismantling objects. Let $\hbar$ be the family of all nonempty subsets of $\overline{1, N}=\{1 ; \ldots ; N\}$. We consider as a route the permutations of positive numbers $\alpha$. These number indices, following the tradition, called the "city", the number 0 is the basis from which unfolds a particular route, the latter estimated cost of the following 


$$
\begin{aligned}
& \pi(\alpha)=c_{0, \alpha(1)}[\overline{1, N}]+\sum_{i=2}^{N} c_{\alpha(i-1), \alpha(i)}[\{\alpha(j): j \in \overline{i, N}\}]= \\
& =c_{0, \alpha(1)}[1, N]+\sum_{i=2}^{N} c_{\alpha(i-1), \alpha(i)}[\overline{1, N} \backslash\{\alpha(j): j \in \overline{1, i-1}\}),
\end{aligned}
$$

This functional characterizes a total radiation dose for the selected route $\alpha$. This route $\alpha$ in this problem satisfies the preceding condition. These conditions give rise to technological constraints on the order of execution. Suppose we have a fixed set from $\hbar$ and each element of which consists of pairs of the form $(p, q)$. Here $p$ is the index of the sender and $q$ is the index of the receiver. Then by $\alpha^{-1}$ we define the permutation in $\overline{1, N}$, that is inverse to $\alpha$. The set of admissible routes has the form

$$
A:=\left\{\alpha \in \hbar \mid \alpha^{-1}(p)<\alpha^{-1}(q)\right\} .
$$

Thus if $\alpha \in A$, then for all pairs $(k, l) \in Q$ and numbers $i$ and $j$ such that $k=\alpha(i), l=\alpha(j)$ the following property holds $i<j$ (this means that the city $l$ is visited after the city $k$ ).

We shall consider the problem:

$$
\pi(\alpha) \rightarrow \min , \alpha \in \mathrm{A} .
$$

For value of the problem (5) we will enter a notation

$$
V:=\min \pi(\alpha), \alpha \in \mathrm{A} \text {. }
$$

Any route $\alpha_{0} \in A$ is called the optimal route, if $\pi\left(\alpha_{0}\right)=V$.

\section{Dynamic programming method}

We have constructed a generalization of the dynamic programming method for the above problem. The Bellman function is built iteratively. First we construct the Bellman function for all singleton sets. Then, for all two-point sets, and so on. In the result, we obtain the Bellman function for the original $N$-point set. The Bellman function gives us the optimal value for the functional (5).

Consider a shortened route problem, defined as the extremum problem similar to (5), but on a visit not all "cities" from $\overline{1, N}$. We introduce the set $\Sigma[K]$ all pairs $(k, l) \in Q$ for which $k \in K$ and $l \in K$. Then $\mathbf{I}[K]$ is the set of all $k \in K$ such that $k \neq q^{\prime}$ for all $\left(p^{\prime}, q^{\prime}\right) \in \Sigma[K][4,5]$. If $K \in \hbar$, then by $|K|$ we denote the number of elements of the set $K$. Let $(b i)[K]$ is the set of all bijections [6] $\alpha$ : $k \neq q^{\prime} \overline{1,|K|} \rightarrow K$. We assume that for every $K \in \hbar$ (I-bi) $[K]$ is the set of all bijections $\alpha \in(b i)[K]$ such that $n=|K|$.

Now we consider a shortened version of the quality criterion (3) 


$$
\begin{gathered}
\pi_{K}^{(i)}(\alpha):=c_{i, \alpha(1)}[K]+\sum_{j=2}^{|K|} c_{\alpha(j-1), \alpha(j)}[\{\alpha(\ell): \ell \in \overline{j,|K|})]= \\
\quad=c_{i, \alpha(1)}[K]+\sum_{j=1}^{|K|-1} c_{\alpha(j-1), \alpha(j)}[\{\alpha(\ell): \ell \in \overline{j+1,|K|})]
\end{gathered}
$$

We introduce the following truncated problem

$$
\pi_{K}^{(i)}(\alpha) \rightarrow \min , \alpha \in(\mathrm{I}-\mathrm{bi})[K] .
$$

It is useful to bear in mind that

$$
\pi_{1, N}^{(0)}(\alpha)=\pi(\alpha) .
$$

Now we introduce the extremes (values) of truncated problems (8): if $K \in \hbar$ and $i \in \overline{0, N}$, then

$$
v(i, K)=\min _{\alpha \in(\mathrm{I}-\mathrm{bi})[K]} \pi_{K}^{(i)}(\alpha) .
$$

From (10) we obtain in particular

$$
V=v(0, \overline{1, N}) .
$$

Finally, we assume by definition that

$$
v(i, \varnothing):=0
$$

for all $i \in \overline{0, N}$. Thus we have defined the Bellman function for the problem (8) $[3,4]$. Bellman equation

$$
v(i, K)=\min _{k \in \mathrm{I}(K)}\left(c_{i, k}[K]+v(k, K \backslash\{k\})\right) .
$$

is valid for the problem (8).

Equation (13) allows you to recursively build all functions $v(i, K)$ As a result, we obtain a value of (11). After that, with the help of $v(i, K)$ we can build the route. Detailed description of the algorithm given in [3-5]

\section{Some simulation results}

Now we consider a model problem of dismantling nuclear power plant unit. The radiation dose received during the dismantlement of $\alpha(i)$ object will be determined as follows:

$$
c_{\alpha(i), \alpha(i+1)}[\alpha(j): j \in \overline{i+1, N]}]=t_{\alpha(i)}\left(H_{\alpha(i)}+\sum_{l=i+1}^{N} \frac{H_{\alpha(l)}}{r_{\alpha(i), \alpha(l)}^{2}}\right),
$$

where $t_{i}$ is the time of dismantling of the i-th element, $H_{i}$ - the intensity of the radiation $\mathrm{i}$-th element. The distance between objects in the model example is given by

$$
r_{i, j}=\frac{(|i-j|+i \times j) \times|i-j|}{i \times j}+1 .
$$

The total dose to be minimized, will be given by 


$$
\pi(\alpha)=t_{\alpha(1)}\left(H_{\alpha(1)}+\sum_{l=2}^{N} \frac{H_{\alpha(l)}}{r_{\alpha(1), \alpha(l)}^{2}}\right)+t_{\alpha(2)}\left(H_{\alpha(2)}+\sum_{l=3}^{N} \frac{H_{\alpha(l)}}{r_{\alpha(2), \alpha(l)}^{2}}\right)+\ldots+t_{\alpha(N)} H_{\alpha(N)} .
$$

We assume that the number of objects to be dismantled is 16 . The radiation intensity in units per hour and a time in hours given in table dismantling.

Table 2: $\quad$ Radiation intensity and the dismantling of facilities.

\begin{tabular}{|l|l|l|l|l|l|l|}
\hline № & 1 & 2 & 3 & 4 & 5 & 6 \\
\hline$t_{i}$ & 1.6 & 2.2 & 0.7 & 3.1 & 2.1 & 0.3 \\
\hline$H_{i}$. & 0.75 & 0.99 & 2.1 & 1.45 & 1.32 & 1.21 \\
\hline № & 9 & 10 & 11 & 12 & 13 & 14 \\
\hline$t_{i}$ & 2.2 & 4.3 & 1.9 & 2.5 & 3.3 & 0.6 \\
\hline$H_{i}$ & 0.63 & 1.72 & 2.22 & 0.94 & 0.32 & 1.76 \\
\hline
\end{tabular}

Furthermore, we assume that the eight pairs set precedence conditions: 1-2, 5$6,9-12,9-13,1-15,1-16,3-5,4-9$. Optimal results in this case are as follows: $3-$ 14-7-5-6-1-15-11-16-4-2-10-8-9-12-13 optimum route, the minimum value of the functional (14) 4640 conventional units.

Software implementation of several dozen examples for various contaminated technological systems consisting of 10-20 members, showed the computational efficiency of the developed method [3-5]. Optimization sequence dismantling leads to a reduction of $25-40 \%$ of the total radiation dose to personnel.

\section{Conclusions}

1. Calculation methods of optimization sequence radioactive equipment dismantling of decommissioned nuclear power units have considerable potential in solving the problem of minimizing exposure to personnel.

2. The dynamic programming method is a very effective tool to solve the problem.

3. Optimization of the sequence of dismantling radioactive objects can reduce the radiation dose by $25-40 \%$.

\section{Acknowledgement}

This study was supported by the Russian Foundation for Basic Research, projects no. 13-08-00643, 13-01-90414 and 13-01-96022. 


\section{References}

[1] ICRP Publication 103. Recommendations of the UCRP. Annals of the ICRP, v. 37/2-4, 2007.

[2] Tashlykov O.L., Sesekin A.N., Shcheklein S.E., Balushkin F.A., Chentsov A.G., Khomyakov A.P. The Mathematical Simulation Methods Capability for Solution of the Personnel Irradiation Decrease Problem // Radiation Safety Problems. 2009. №4. pp. 47-57.

[3] Sesekin A.N., Chentsov A.A., Chentsov A.G. A Generalized Courier Problem with Cost Function Depending on the List of Tasks. Journal of Computer and Systems Sciences International, 2010, Vol. 49, No. 2, pp. 234-243.

[4] Chentsov A.G., Sesekin A.N., Shcheklein S.Y., Tashlykov O.L. On One Modification of Traveling Salesman Problem Oriented on Application in Atomic Engineering // American Institute of Physics. Conference Proceeding, Vol. 1293, 2010, pp. 197-202.

[5] Tashlykov O.L., Sheklein S.Ye., Sesekin A.N. The route optimization of radiation-dangerous works // Odeskyi Politechnichnyi Universytet. Pratsi: Scientific, science and technology collected articles. - Odesa, 2012. - Iss. 1(38). pp. 118-125.

[6] Cormen T.H., Leiserson C.E., Rives, R.L. Introduction to Algorithms. 1st. - MIT Press and McGraw-Hill, 1990. 